\title{
SUNSPOT AND STARSPOT LIFETIMES IN A TURBULENT EROSION MODEL
}

\author{
Yuri E. LitVinenko ${ }^{1}$ and M. S. Wheatland ${ }^{2}$ \\ ${ }^{1}$ Department of Mathematics, University of Waikato, P. B. 3105, Hamilton, New Zealand \\ ${ }^{2}$ Sydney Institute for Astronomy, School of Physics, The University of Sydney, NSW 2006, Australia \\ Received 2016 September 26; revised 2016 November 11; accepted 2016 November 11; published 2017 January 6
}

\begin{abstract}
Quantitative models of sunspot and starspot decay predict the timescale of magnetic diffusion and may yield important constraints in stellar dynamo models. Motivated by recent measurements of starspot lifetimes, we investigate the disintegration of a magnetic flux tube by nonlinear diffusion. Previous theoretical studies are extended by considering two physically motivated functional forms for the nonlinear diffusion coefficient $D$ : an inverse power-law dependence $D \propto B^{-\nu}$ and a step-function dependence of $D$ on the magnetic field magnitude $B$. Analytical self-similar solutions are presented for the power-law case, including solutions exhibiting "superfast" diffusion. For the step-function case, the heat-balance integral method yields approximate solutions, valid for moderately suppressed diffusion in the spot. The accuracy of the resulting solutions is confirmed numerically, using a method which provides an accurate description of long-time evolution by imposing boundary conditions at infinite distance from the spot. The new models may allow insight into the differences and similarities between sunspots and starspots.
\end{abstract}

Key words: diffusion - stars: magnetic field - starspots - Sun: magnetic fields - sunspots - turbulence

\section{INTRODUCTION}

A great deal of effort has gone into observing and analyzing the disintegration of sunspots (and starspots). Sunspot decay is usually characterized by the rate of decrease of the sunspot area $A$, and numerous observations appear to be consistent with a parabolic decay law, with $A(t)$ a decreasing quadratic function of time $t$ (e.g., Moreno-Insertis \& Vázquez 1988; Martínez Pillet et al. 1993; Petrovay \& van Driel-Gesztelyi 1997).

Early theories invoked the turbulent diffusion of the magnetic field within the spot to model the observed rate of decay, yet such models predicted a linear decay law, corresponding to a constant area decay rate $d A / d t$ (Meyer et al. 1974; Krause \& Rüdiger 1975). In order to explain a parabolic decay, Petrovay \& Moreno-Insertis (1997) developed a model of sunspot disintegration by turbulent "erosion" of penumbral boundaries, which occurs when bits of the magnetic field are sliced away from the edge of a sunspot and swept to the supergranular cell boundaries by supergranular flows (Simon \& Leighton 1964).

A key feature of the erosion model is that turbulent diffusivity, associated with the flows, is suppressed within the spot. The assumption is justified by the theoretical prediction that diffusivity $D$ should rapidly decrease if the magnetic field $B$ exceeds an energy equipartition value (Kitchatinov et al. 1994; Rüdiger \& Kitchatinov 2000), which is why diffusivity in the turbulent erosion model may be assumed to be a decreasing function of the magnetic field strength. Petrovay \& van Driel-Gesztelyi (1997) presented observational evidence in support of the parabolic decay law and its theoretical explanation by turbulent erosion.

Litvinenko \& Wheatland (2015) recently revisited the theory of sunspot decay by turbulent erosion, considered as a moving boundary problem. While some of the earlier results were confirmed for moderate sunspot magnetic field strengths, the new analytical and numerical solutions yielded a significantly improved theoretical description of sunspot disintegration. In particular, the dependence of the spot area was shown to be a nonlinear function of time, which in a certain parameter regime can be approximated by a parabola. More accurate expressions for the spot lifetime in terms of an initial magnetic field were derived analytically and verified numerically.

Following Petrovay \& Moreno-Insertis (1997), Litvinenko \& Wheatland (2015) assumed in their study that the turbulent diffusivity $D=D(B)$ within a decaying sunspot is much less than that outside the spot. A more realistic model should incorporate a more realistic dependence of the turbulent diffusivity on the field strength within the spot. Our aim in this paper is to further develop the theory of turbulent erosion by exploring the effect of a non-vanishing diffusivity within a sunspot on the rate of its disintegration.

Theoretical mechanisms and observable features of the diffusive transport of the photospheric magnetic field have been a subject of intense research activity (e.g., Chatterjee et al. 2006; Litvinenko 2011; Lepreti et al. 2012; Rempel \& Cheung 2014). Our detailed analysis of a simple nonlinear model, reinforced by numerical solutions, can complement more detailed magnetohydrodynamic simulations (e.g., Hurlburt \& DeRosa 2008; Rempel \& Cheung 2014) and guide empirical models (Gafeira et al. 2014) in studies of sunspot and starspot evolution. The determination of the lifetimes of spots is a topic of general-and current-astrophysical interest. Quantitative models for starspot evolution may help estimate the magnetic diffusion timescale and thus yield important constraints in stellar dynamo models (e.g., Bradshaw \& Hartigan 2014; Davenport et al. 2015; Künstler et al. 2015)-which provides further motivation for our exploration of the turbulent erosion model.

\section{FORMULATION OF THE PROBLEM}

Following Petrovay \& Moreno-Insertis (1997) and Litvinenko \& Wheatland (2015), we model a decaying sunspot as a cylindrically symmetric flux tube. The evolution of the magnetic field $\boldsymbol{B}=B(r, t) \hat{z}$ is governed by the following nonlinear diffusion equation:

$$
\frac{\partial B}{\partial t}=\frac{1}{r} \frac{\partial}{\partial r}\left(r D \frac{\partial B}{\partial r}\right)
$$

where $t$ is the time and $r$ is the distance from the $z$-axis. 
Turbulent diffusivity $D$ is suppressed in a magnetic field exceeding an energy equipartition value $B_{e}$ (Kitchatinov et al. 1994), where $B_{e} \simeq 400 \mathrm{G}$ for typical parameters of the solar photosphere (Petrovay \& Moreno-Insertis 1997). It follows that $D$ is a decreasing function of $B$, although the exact functional dependence remains uncertain. Below we investigate several choices for $D=D(B)$, which yield analytical solutions.

As previously (Litvinenko \& Wheatland 2015), we choose dimensionless units so that $B_{e}=r_{0}=D_{0}=1$, where $D_{0}=D$ $\left(B_{e}\right)$ and $r_{0}$ is the initial radius of the flux tube. Consequently, the time is measured in units of $r_{0}^{2} / D_{0}$. The initial-value problem is specified by the dimensionless magnetic field profile $B(r, 0)$ at $t=0$, where

$$
B(0,0)=B_{0}
$$

is the maximum field within the spot. Another important parameter is the total magnetic flux of the spot

$$
\Phi_{0}=2 \pi \int_{0}^{\infty} B r d r
$$

An initially present sunspot corresponds to $B_{0}>1$ (or $B_{0}>B_{e}$ in dimensional units). In the following we define the dimensionless flux tube radius $r_{e}(t)$ by the condition that

$$
B\left(r_{e}, t\right)=1
$$

at its edge. Our choice of having the length scale $r_{0}$ be the initial radius of the spot means that

$$
r_{e}(0)=1,
$$

and it follows that

$$
B(1,0)=1 \text {. }
$$

Finally, we define the spot decay time $T$ by the condition

$$
r_{e}(T)=0
$$

which is equivalent to

$$
B(0, T)=1 .
$$

\section{EXACT SELF-SIMILAR SOLUTIONS FOR NONLINEAR DIFFUSION OF A MAGNETIC FLUX TUBE}

Similarity solutions to partial differential equations have a large number of applications (e.g., Barenblatt 1996). In particular self-similar solutions to nonlinear diffusion equations have been considered for several forms of the diffusion coefficient (e.g., King 1990). These similarity reductions not only lead to exact solutions of specific initial-value problems but also serve as intermediate asymptotics that approximate solutions of a much larger class of problems. Here we consider a similarity reduction of the nonlinear two-dimensional diffusion Equation (1), assuming that the dimensionless diffusion coefficient has a power-law form of

$$
D(B)=B^{-\nu},
$$

where we take $\nu>0$ in order to model the suppression of magnetic diffusivity in the strong magnetic field of a sunspot.

The self-similar solution to Equation (1), which satisfies the flux conservation condition $\Phi_{0}=$ const, is known to take the form

$$
B(r, t)=\left(t_{0}+t\right)^{-1 /(1-\nu)} \phi(\xi), \quad \xi=r\left(t_{0}+t\right)^{-1 / 2(1-\nu)}
$$

with $t_{0}=$ const (Barenblatt 1952; Pattle 1959). On substituting this form into Equation (1), solving for $\phi(\xi)$, and using Equation (3) to specify an integration constant, we get the following expression for an evolving field profile:

$$
\begin{aligned}
B(r, t)= & \left(t_{0}+t\right)^{-1 /(1-\nu)}\left[\left(\frac{4 \pi}{\Phi_{0}}\right)^{\nu /(1-\nu)}\right. \\
& \left.+\frac{\nu}{4(1-\nu)}\left(t_{0}+t\right)^{-1 /(1-\nu)} r^{2}\right]^{-1 / \nu} .
\end{aligned}
$$

It follows that the maximum field (at $r=0$ ) is given by

$$
B(0, t)=\left(\frac{\Phi_{0}}{4 \pi\left(t_{0}+t\right)}\right)^{1 /(1-\nu)},
$$

and so

$$
B_{0}=\left(\frac{\Phi_{0}}{4 \pi t_{0}}\right)^{1 /(1-\nu)} .
$$

Now Equation (6) yields the magnetic flux

$$
\Phi_{0}=\frac{\pi \nu}{(1-\nu)} \frac{B_{0}}{B_{0}^{\nu}-1} .
$$

To find the decay time $T$ for a given initial magnetic field $B_{0}$, we express the parameter $t_{0}$ in terms of $B_{0}$ and substitute the resulting expression into Equation (12). On solving Equation (8) for $T$ and using Equation (14) to eliminate $\Phi_{0}$, we obtain the sunspot decay time in terms of the parameters $B_{0}$ and $\nu$ :

$$
T=\frac{\nu}{4(1-\nu)} \frac{B_{0}-B_{0}^{\nu}}{B_{0}^{\nu}-1} .
$$

In the limit $\nu \rightarrow 0$, Equation (15) reduces to the expression for the case of linear diffusion:

$$
\left.T\right|_{\nu=0}=\frac{B_{0}-1}{4 \ln B_{0}} .
$$

Note that the self-similar solution has a curious feature: Equation (15) predicts that $T \rightarrow 1 / 4$ as $B_{0} \rightarrow 1$ (for any value of $\nu$ ), whereas it is physically obvious that $T=0$ when $B_{0}=1$. This singular limit behavior is related to the fact that the magnetic flux $\Phi_{0} \rightarrow \infty$ as $B_{0} \rightarrow 1$. In practice this does not cause any problems since we always assume the initial field $B_{0}>1$ in order to model a sunspot.

The self-similar solution above is applicable only for $\nu<1$ since the flux integral in Equation (3) diverges for larger values of $\nu$, physically corresponding to an instantaneous flux transfer to infinity (Landau \& Lifshitz 1987). More generally, $\nu<2 / N$ is required to avoid the divergence of the flux integral for diffusion in $N$ dimensions. Mathematical issues of the existence and uniqueness of solutions were analyzed by Brezis \& Friedman (1983).

While solutions for $\nu \geqslant 1$ formally violate the total flux conservation, they are mathematically correct and may provide a useful local description of nonlinear diffusion. As an illustration, consider the case $\nu=1$. It is straightforward to 
derive a separable solution to Equation (1). By assuming $B(r, t)=(T-t) F(r)$, we reduce the problem to a secondorder ordinary differential equation for the spatial part $F(r)$. The solution is as follows:

$$
F(r)=\frac{8 c_{0}}{\left(c_{0}+r^{2}\right)^{2}},
$$

where $c_{0}$ is an integration constant and the other integration constant is determined by the requirement that $F$ be finite at $r=0$. In a different context, Equation (17), in particular, case $c_{0}=1$, was given by Rosenau (1995).

On using Equations (2) and (3) to express $T$ and $c_{0}$ in terms of $B_{0}$ and $\Phi_{0}$, we obtain

$$
\frac{B(r, t)}{B_{0}}=\left(1+\frac{\pi B_{0} r^{2}}{\Phi_{0}}\right)^{-2}\left(1-\frac{8 \pi t}{\Phi_{0}}\right) .
$$

Here $\Phi_{0}=\Phi(0)$ is the initial magnetic flux of the spot (at $t=0$ ), which decreases with time according to

$$
\Phi(t)=\Phi_{0}-8 \pi t .
$$

The localized magnetic field profile is seen to shrink with time until the spot vanishes at

$$
T=\frac{\Phi_{0}}{8 \pi} .
$$

The termination of the process in finite time was referred to by Rosenau (1995) as "superfast" diffusion. This unusual feature of the solution is related to the singular behavior of $D(B)=1$ / $B$ as $B \rightarrow 0$ : in sharp contrast to the solution of a linear problem, the continuity flux

$$
\begin{aligned}
\mathcal{F} & =-2 \pi r D \frac{\partial B}{\partial r} \\
& =\frac{8 \pi^{2} r^{2}}{\Phi_{0}+\pi B_{0} r^{2}}
\end{aligned}
$$

is independent of time and approaches a constant value, $\mathcal{F} \rightarrow 8 \pi / B_{0}$, as $r \rightarrow \infty$, which results in "flux suction at infinity" (Rosenau 1995). Physically, because $B \rightarrow 0$ as $t \rightarrow T$, the diffusivity $D \rightarrow \infty$. Consequently, the diffusion timescale $1 / D \rightarrow 0$, and so a diffusive description breaks down as $t \rightarrow T$.

\section{SOLUTIONS FOR THE CASE OF MODERATELY SUPPRESSED MAGNETIC DIFFUSIVITY WITHIN A SPOT}

We now consider a different model for diffusivity suppression (quenching) within a spot, which complements the analysis by Litvinenko \& Wheatland (2015). We assume that the evolution of the magnetic field is described by the nonlinear two-dimensional diffusion Equation (1) with a step dependence in the dimensionless diffusion coefficient:

$$
D=1, \quad B<1
$$

and

$$
D=1-\epsilon, \quad B>1 .
$$

Litvinenko \& Wheatland (2015) considered the case $\epsilon=1$, which corresponds to a very strong suppression of turbulent diffusivity within the spot. A more realistic model would correspond to a less severe turbulent suppression of the diffusivity within the spot. Therefore, we generalize our nonlinear diffusion model to incorporate the effect of a nonvanishing diffusivity within a sunspot on the rate of its disintegration.

To obtain an analytical solution for the case of a moderate diffusivity suppression within the spot, we use the heat-balance integral method (Goodman 1958), which proved to yield accurate approximations in problems of nonlinear diffusion (Hill \& Dewynne 1987; Barenblatt 1996). The basic idea is to require that an approximate solution satisfy an integral of a nonlinear equation rather than the equation itself. We apply the method to describe the turbulent erosion of a sunspot, modeled as a cylindrically symmetric flux tube.

We assume that $0<\epsilon \ll 1$ and seek an approximate solution of the form

$$
B(r, t)=f(t) \exp \left[-g(t) r^{2}\right] .
$$

The flux tube radius $r_{e}(t)$ is defined by Equation (4). Consequently, we have

$$
f=\exp \left(g r_{e}^{2}\right) .
$$

Integration of Equation (1) over $r$ from 0 to $\infty$ and substitution of the self-similar form (24) into the resulting equation yields

$$
\frac{d}{d t}\left[\frac{\exp \left(g r_{e}^{2}\right)}{g}\right]=4 \epsilon g r_{e}^{2},
$$

where Equation (4) was used to simplify the right-hand side and Equation (25) was used to eliminate $f(t)$.

In the linear case $\epsilon=0$, the solution of the initial-value problem with a Gaussian profile at $t=0$ is given by

$$
B(r, t)=\frac{B_{0}}{1+4 t \ln B_{0}} \exp \left(-\frac{r^{2} \ln B_{0}}{1+4 t \ln B_{0}}\right) .
$$

Here the parameters of an evolving Gaussian profile are chosen to satisfy the initial conditions, given by Equations (2) and (6).

The decay time, defined by Equations (7) and (8), is easily shown to be

$$
\left.T\right|_{\epsilon=0}=\frac{B_{0}-1}{4 \ln B_{0}},
$$

where, as previously, we assume $B_{0}>1$ in order to exclude solutions with an infinite magnetic flux. Note for clarity that if $B_{0}>e$, we have $d r_{e} / d t>0$ at $t=0$ in the linear solution, and so diffusion causes the flux tube radius to increase until it reaches a maximum at $t=\left(B_{0} / e-1\right) / 4 \ln B_{0}$ and then to decrease.

Motivated by the form of the linear solution, we substitute

$$
g(t)=\frac{\ln B_{0}}{1+4 t \ln B_{0}}
$$

into the approximate Equation (24), describing a weakly nonlinear case $0<\epsilon \ll 1$. Thus, Equation (26) becomes an ordinary differential equation for the flux tube radius $r_{e}(t)$, which should be solved subject to the initial condition given by Equation (5). Equation (7) then yields the spot decay time $T$.

Keeping in mind that $\epsilon$ is assumed to be small, we solve Equation (26) by iteration. On substituting a simple linear 
function

$$
r_{e}^{2} \approx 1-\frac{t}{T}
$$

into the right-hand side of Equation (26) and integrating from 0 to $t$, we get an approximate analytical expression for $r_{e}^{2}$ :

$$
\begin{aligned}
r_{e}^{2} \approx & \frac{1+4 t \ln B_{0}}{\ln B_{0}} \ln \left\{\frac{B_{0}}{1+4 t \ln B_{0}}+\epsilon \frac{\ln B_{0}}{1+4 t \ln B_{0}}\right. \\
& \left.\times\left[\left(1+\frac{1}{4 T \ln B_{0}}\right) \ln \left(1+4 t \ln B_{0}\right)-\frac{t}{T}\right]\right\} .
\end{aligned}
$$

On setting $r_{e}^{2}(T)=0$, we obtain an algebraic equation for the decay time $T$ :

$$
\begin{aligned}
& \frac{B_{0}}{1+4 T \ln B_{0}}+\epsilon \frac{\ln B_{0}}{1+4 T \ln B_{0}}\left[\left(1+\frac{1}{4 T \ln B_{0}}\right)\right. \\
& \left.\times \ln \left(1+4 T \ln B_{0}\right)-1\right]=1 .
\end{aligned}
$$

Alternatively, integration of Equation (26) over $t$ from 0 to $T$ yields an expression for $T$, which makes clear that $\epsilon>0$ leads to a slower decay:

$$
T=\frac{B_{0}-1}{4 \ln B_{0}}+\epsilon \int_{0}^{T} g r_{e}^{2} d t
$$

Now substitution of Equations (29) and (30) into the right-hand side of Equation (33) yields Equation (32).

To solve Equation (32) in the case of a small $\epsilon$, we replace $T$ by $\left.T\right|_{\epsilon=0}$ from Equation (28) in all terms containing $\epsilon$. The result is as follows:

$$
T \approx \frac{B_{0}-1}{4 \ln B_{0}}+\frac{\epsilon}{4}\left[\left(1+\frac{1}{B_{0}-1}\right) \ln B_{0}-1\right] .
$$

For a fixed $\epsilon \geqslant 0, T=T\left(B_{0}\right)$ is an increasing function of $B_{0}$.

We note that the assumed form for the magnetic diffusivity $D$, given by Equations (22) and (23), is finite in the limiting case $B \rightarrow \infty$, while the real magnetic diffusivity is expected to vanish in this limit. However, since $B(r, t)$ remains finite in our solution for any $t>0$, the behavior of $D$ as $B \rightarrow \infty$ is irrelevant. Our assumption of a suppressed but nonzero diffusivity inside the spot is physically meaningful as long as the magnetic field $B$ remains finite, as it does in our solution. We have previously considered a solution for $B(r, t)$ valid when the diffusivity vanishes within the spot (Litvinenko \& Wheatland 2015), which is accurate for a very strong magnetic field $B_{0}$ in the spot. The new solution presented here quantifies the effects of a non-vanishing $D$.

\section{NUMERICAL SOLUTION}

To quantify the accuracy of the analytical results, Equation (1) is solved numerically. We use an explicit scheme which maps the region $[0, \infty]$ in radius $r$ to the region $[0,1]$ in a transformed independent variable $x$, as explained in the Appendix. The approach allows an exact boundary condition to be imposed at $r=\infty$, which provides more accurate solutions than an approximate boundary condition at a finite radius. We present solutions with a grid spacing $h=0.01$ in $x$ and with a time step one quarter of the stability limit identified in the Appendix.
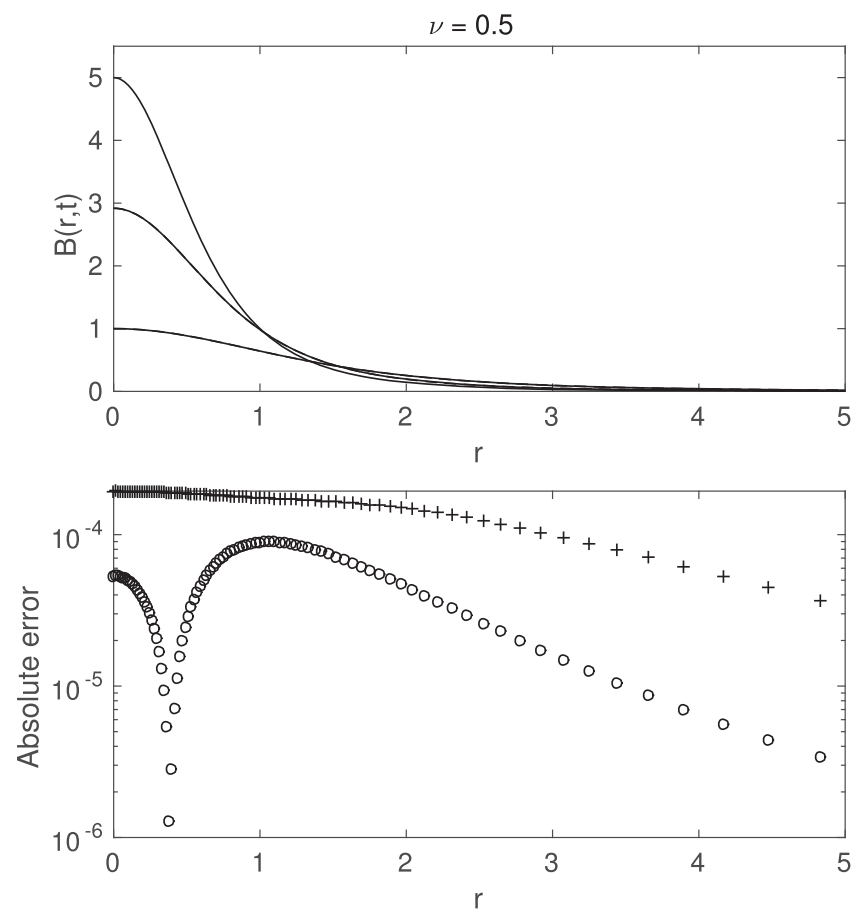

Figure 1. Solution to Equation (1) for the case $D(B)=B^{-\nu}$ with $\nu=0.5$ and $B_{0}=5$. The upper panel shows the analytic (solid) and numerical (dashed) solutions at $t=0, t=\frac{1}{4} T$, and $t=T$. The numerical solutions coincide with the analytic solutions and are not visible. The lower panel plots the absolute error in the numerical solution at $t=\frac{1}{4} T$ (circles) and $t=T$ (plus signs).

\subsection{Solutions for the Exact Self-similar Case}

To test the numerical method and to illustrate the properties of the analytic solutions presented in Section 3, we consider a solution with the power-law form for the diffusion coefficient, Equation (9), which admits self-similar solutions.

First, we consider a flux-conserving case $(\nu=0.5)$, with $B_{0}=5$. The solid curves in the upper panel of Figure 1 show the analytic magnetic field profile $B(r, t)$ given by Equation (11) at three times during the spot evolution $t=0, t=\frac{1}{4} T$, and $t=T$, where $T$ is the decay time defined by Equation (15). The numerical solutions are also shown in this panel by dashed curves, but they coincide with the solid curves and are not visible. The lower panel shows the absolute error in the numerical solution at times $t=\frac{1}{4} T$ (circles) and $t=T$ (plus signs). The maximum error is $\approx 2 \times 10^{-4}$. The numerical solution conserves flux throughout the time evolution $\left(3.2 \times 10^{4}\right.$ time steps) to within $0.11 \%$.

Second, we consider a non-flux-conserving case $\nu=1$, which exhibits superfast diffusion, i.e., the vanishing of the spot at the time $T$ defined by Equation (20). The upper panel of Figure 2 shows the analytic and numerical solutions at three times $t=0, t=\frac{1}{4} T$, and $t=T$ for the case $B_{0}=\Phi_{0}=1$, with a log-linear display used to illustrate the behavior for large $r$. The analytic solutions are the solid curves, and the numerical solutions at times $t=\frac{1}{4} T$ and $t=T$ are shown as circles and plus signs, respectively. The analytic solution at time $t=T$ is identically zero. The numerical solution is accurate initially, but becomes inaccurate as $t \rightarrow T$. The reason for the error is that the boundary condition at infinity, Equation (45), does not reproduce the behavior of the continuity flux at large $r$, as shown in the lower panel. In particular the numerical solution 

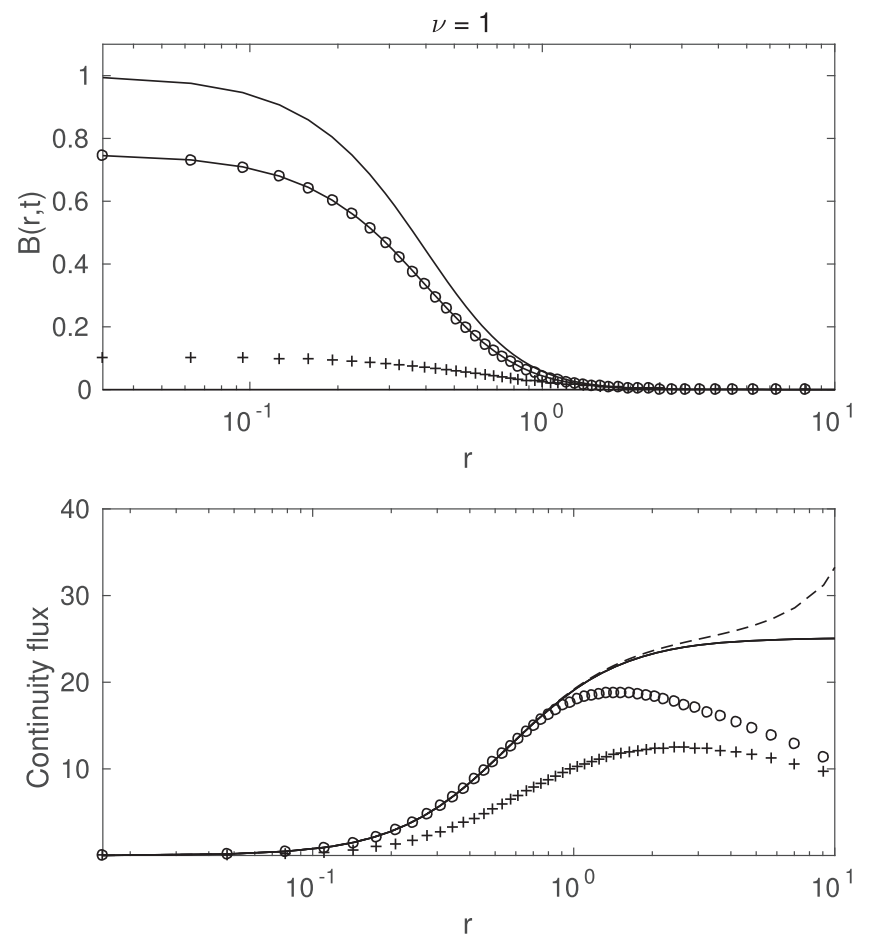

Figure 2. Solution to Equation (1) for the case $D(B)=B^{-\nu}$ with $\nu=1$, an example of superfast diffusion. The upper panel shows the field profile $B(r, t)$ at times $t=0, t=\frac{1}{4} T$, and $t=T$ for $B_{0}=\Phi_{0}=1$. The analytic solutions are the solid curves, and the numerical solutions are shown as circles and plus signs at times $t=\frac{1}{4} T$ and $t=T$. At time $t=T$ the analytic solution is zero. The lower panel plots the continuity flux, which is independent of time in the analytic solution (solid curve). The continuity flux in the numerical solution is shown as a dashed curve $(t=0)$, circles $\left(t=\frac{1}{4} T\right)$, and plus signs $(t=T)$. A log-linear display is used to show the behavior for large $r$.

does not maintain a large positive value of the continuity flux as $r \rightarrow \infty$, which produces "flux suction at infinity." This example demonstrates the need to accurately represent the boundary conditions at large $r$ in the solutions.

\subsection{Solutions for the Case of a Moderate Suppression of $D(B)$ within a Spot}

The numerical solution for the case with a diffusion coefficient defined by Equations (22) and (23) provides a test of the heat-balance integral method results presented in Section 4 and in particular of expression (34) for the lifetime. In this case we do not have an exact solution to ensure accuracy, but the numerical solutions presented here conserve flux during the time evolution to within $4 \times 10^{-4} \%$ in the worst case.

First, we consider the field profile $B(r, t)$ for the heat-balance solution, specified by Equations (24), (25), (29), and (31). Figure 3 compares the heat-balance method profiles (solid curves) with the numerical solutions (dashed curves) at the three times $t=0, t=\frac{1}{4} T$, and $t=T$. The solutions assume an initial magnetic field strength $B_{0}=5$. The left panel shows the case $\epsilon=0.25$, and the right panel shows the case $\epsilon=0.5$. For the smaller value of $\epsilon$ the heat-balance integral method provides a good approximation to the field profile throughout the evolution. The method is somewhat less accurate for the larger value of $\epsilon$.
Figure 4 repeats the display in Figure 1, but shows the case $B_{0}=10$. The accuracy of the field profile obtained with the heat-balance integral method is not strongly dependent on the choice of $B_{0}$.

Second, we consider the accuracy of the heat-balance integral estimate for the spot lifetime, Equation (34). The estimate has the form

$$
T \approx T_{0}+\epsilon T_{1},
$$

where $T_{0}$ is the lifetime for the linear case $(\epsilon=0)$, given by Equation (28). Figure 5 compares Equation (35) (solid curves) with the numerical solution (circles) as a function of $B_{0}$ for the range $2 \leqslant B_{0} \leqslant 10$. The upper panel shows $T$ for the three cases $\epsilon=0, \epsilon=0.25$, and $\epsilon=0.5$ (bottom to top), and the lower panel shows $T-T_{0}$ for the nonlinear cases $\epsilon=0.25$ and $\epsilon=0.5$. Figure 5 demonstrates that the heat-balance method provides a good approximation to the lifetime of the spot for the choice $\epsilon=0.25$ over the range of initial field strengths considered. The approximation is worse for the larger value of $\epsilon$, as expected. It is interesting to consider replacing Equation $(35)$ with the $(0,1)$ Padé approximant:

$$
T \approx \frac{T_{0}}{1-\epsilon T_{1} / T_{0}} .
$$

The dotted curves in Figure 5 show the lifetimes given by Equation (36), and the results show that the Padé expression provides a better approximation for the lifetime. This might be expected based on the final steps in the derivation of Equation (34).

\section{DISCUSSION}

The physical mechanism of sunspot erosion was proposed by Simon \& Leighton (1964). Petrovay \& Moreno-Insertis (1997) introduced a nonlinear diffusion equation of a magnetic flux tube as a model of turbulent erosion. Petrovay \& MorenoInsertis (1997) identified the maximum magnetic field $B_{0}$ in a sunspot as the key parameter that determines the lifetime $T$ of the spot and derived a law of sunspot decay due to turbulent erosion. As Litvinenko \& Wheatland (2015) demonstrated, however, the accuracy of the predictions was limited; for instance, Litvinenko \& Wheatland (2015) have shown that the sunspot lifetime in the model is about half of that originally predicted.

We have presented in this paper a further development of the quantitative theory of sunspot disintegration by turbulent erosion. Our analysis makes it clear that a variety of scalings $T=T\left(B_{0}\right)$ are theoretically possible, depending on initial conditions and the dependence of the turbulent diffusivity on the magnetic field strength within the spot. The results may have implications for the use of the model for estimating the magnetic diffusion timescale in starspots (e.g., Bradshaw \& Hartigan 2014; Davenport et al. 2015; Künstler et al. 2015), which is an important parameter in stellar dynamo models.

Experimental studies typically attempt to infer anomalous, turbulent-driven magnetic diffusivity by equating the lifetime of a spot to a theoretical diffusion timescale (Bradshaw \& Hartigan 2014). We expect that the more detailed analytical models we have developed may help to improve the accuracy of the procedure. More generally, we expect that the new models may allow insight into the differences and similarities 

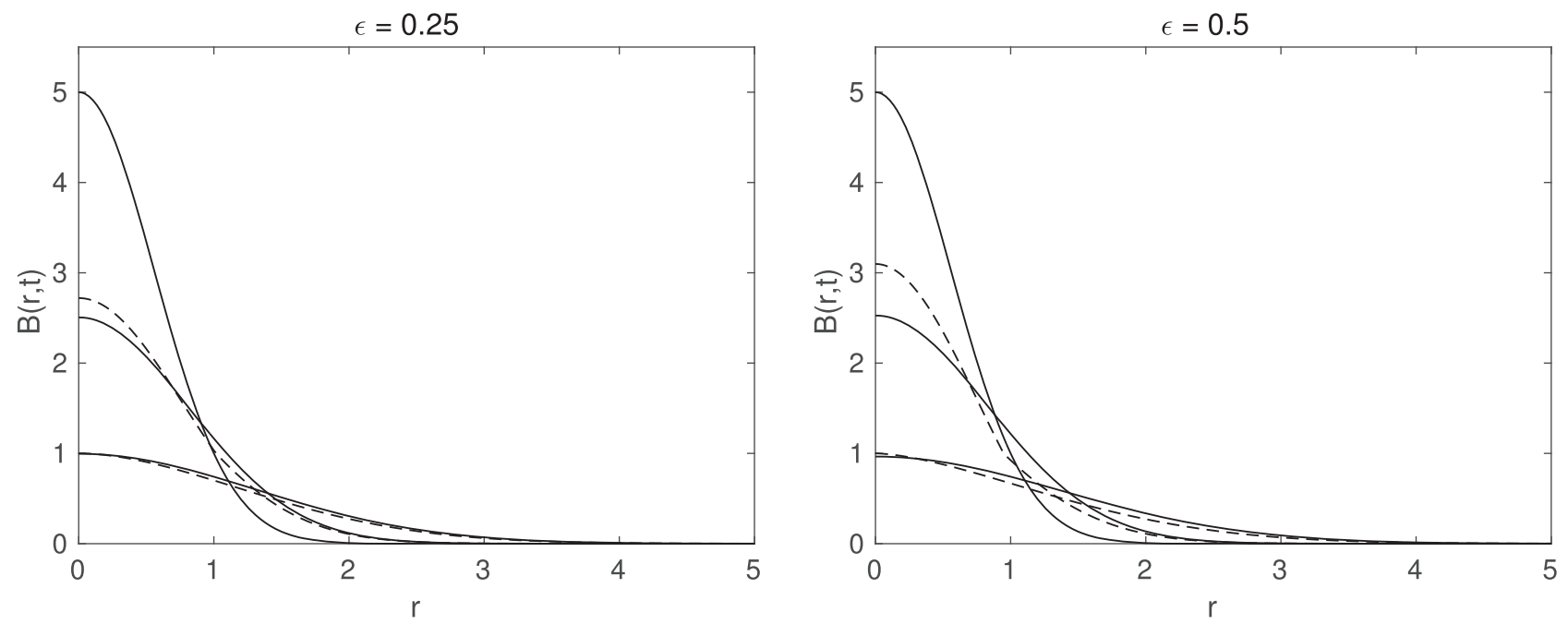

Figure 3. Comparison of the heat-balance integral solution (solid) and the numerical solution (dashed) at $t=0, t=\frac{1}{4} T$, and $t=T$ for a spot with $B_{0}=5$. The left panel is the case $\epsilon=0.25$, and the right panel is the case $\epsilon=0.5$.
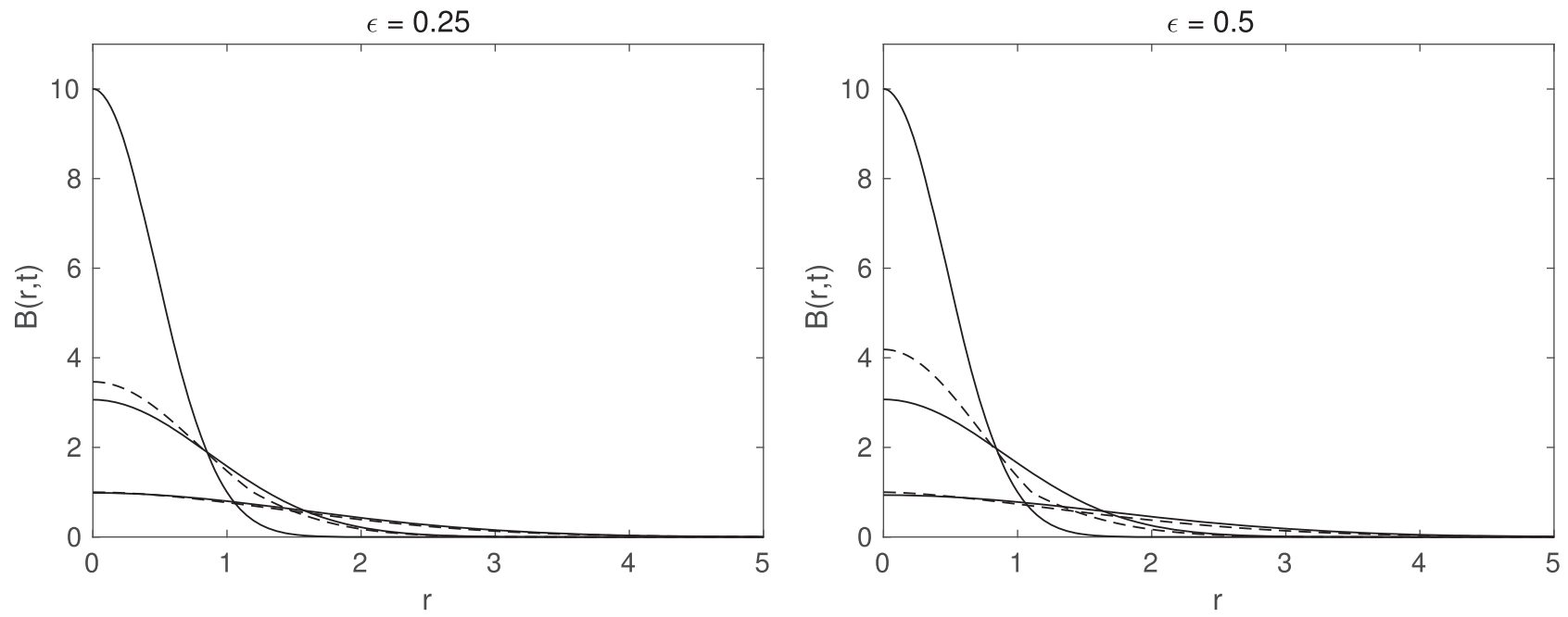

Figure 4. Comparison of the heat-balance integral solution (solid) and the numerical solution (dashed) at $t=0, t=\frac{1}{4} T$, and $t=T$ for a spot with $B_{0}=10$. The left panel is the case $\epsilon=0.25$, and the right panel is the case $\epsilon=0.5$.

between sunspots and starspots. The original formulation of the turbulent erosion model predicted a parabolic dependence of the sunspot area on time (Petrovay \& Moreno-Insertis 1997). Yet deviations from the parabolic decay are large for any one spot (Gafeira et al. 2014), motivating the use of continuous linear piecewise functions in the modeling of starspot growth and decay (Giguere et al. 2016). Such an approach is consistent with the more general decay laws predicted in our analysis, ultimately controlled by the dependence of the magnetic diffusivity on the magnetic field strength within a spot. As pointed out by the referee, however, apparent deviations from the parabolic decay law for individual spots can be caused by the difficulty of identifying individual spots within a decaying sunspot group or by a varying external plage field outside the spots (Petrovay et al. 1999).

The turbulent erosion model can be further improved. Recall that the solution for $\epsilon=1$ in Litvinenko \& Wheatland (2015) describes the turbulent erosion of a sunspot as a moving boundary problem in which the rate of sunspot decay is controlled by the inward speed of a current sheet around the spot. In other words, the decay rate is determined by the local diffusion rate of the magnetic field within the sheet, modeled as a tangential discontinuity at $r=r_{e}(t)$. By contrast, the new solution given by Equations (31) and (34) describes the sunspot decay as determined by global magnetic field diffusion, and field discontinuity is ignored in the smooth profile of the evolving magnetic field, which should be a reasonable assumption if $0<\epsilon \ll 1$. The diffusive evolution of exact self-similar solutions is also a global process. Yet, physically, the sunspot disintegration rate is likely to be influenced by both mechanisms, and so a more accurate solution for intermediate values of $\epsilon$ should incorporate both local and global diffusion processes by considering a more general initial field profile in the spot and more realistic diffusivity dependence on the magnetic field strength. More detailed models of spot decay should also quantify the effects of flux cancelation, which is caused by photospheric magnetic reconnection (e.g., Litvinenko 2015). Application of the model to the data on sunspot and starspot decay may shed light on the physics of turbulent diffusion in magnetized astrophysical plasma. 
The Astrophysical Journal, 834:108 (8pp), 2017 January 10
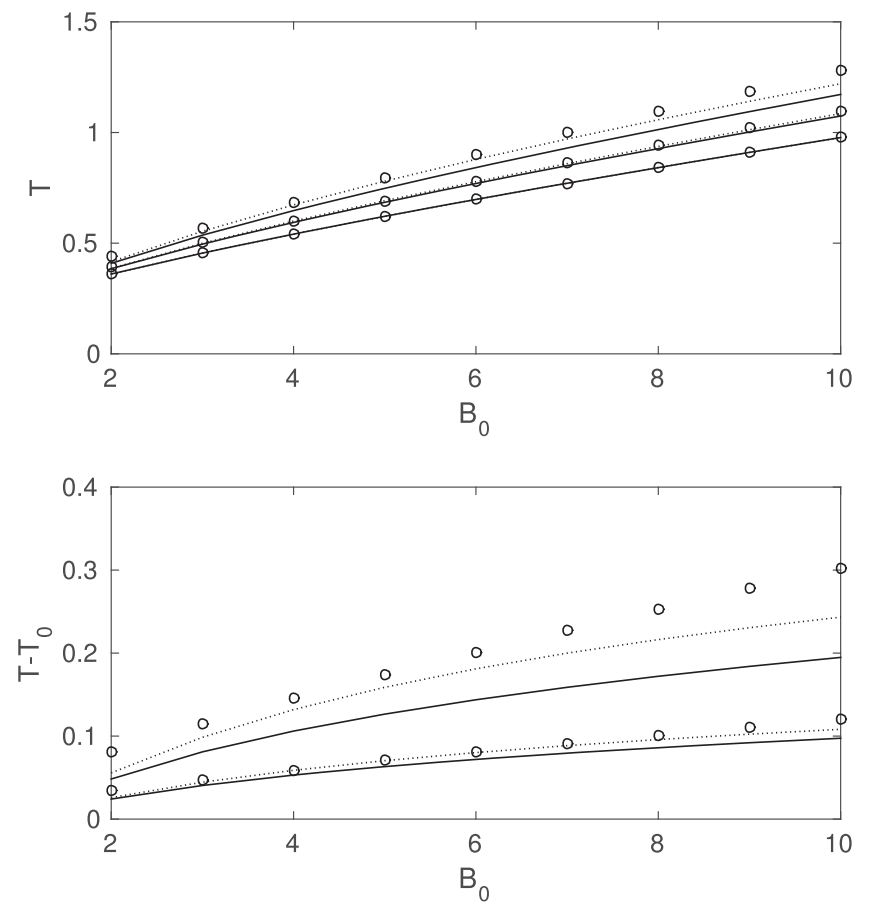

Figure 5. The heat-balance integral estimates (solid) for the spot lifetime and the numerically determined lifetime (circles), plotted as functions of $B_{0}$. The upper panel shows the lifetime $T$, and the lower panel shows $T-T_{0}$, where $T_{0}$ is the linear result $(\epsilon=0)$. In the upper panel, results are shown for $\epsilon=0$, $\epsilon=0.25$, and $\epsilon=0.5$, and the lower panel shows $\epsilon=0.25$ and $\epsilon=0.5$. The dotted curves show the results for the heat-balance integral method using the Padé approximant form for the lifetime.

An anonymous referee's comments and suggestions are gratefully acknowledged.

\section{APPENDIX NUMERICAL METHOD}

Litvinenko \& Wheatland (2015) numerically solved Equation (1) using a Crank-Nicolson scheme. The method imposed a boundary condition at a finite outer boundary $r=r_{m}$, which allowed flux transport across the boundary, using one-sided spatial derivatives. This approach was an improvement over the assumption of zero flux at an outer radius used by Petrovay \& Moreno-Insertis (1997), but it was still a source of error for the long-time integrations necessary to determine the lifetimes for the model spots. Here we use a simpler explicit scheme, but with a transformation of the infinite $r$-domain to a finite domain, to allow an exact outer boundary condition.

The change of independent variable

$$
r=\tan \left(\frac{\pi}{2} x\right)
$$

maps $0 \leqslant r \leqslant \infty$ to $0 \leqslant x \leqslant 1$. Transforming the derivatives in Equation (1) gives

$$
\frac{\partial B}{\partial t}=\frac{4}{\pi^{2} r\left(1+r^{2}\right)} \frac{\partial}{\partial x}\left(\frac{r}{1+r^{2}} D \frac{\partial B}{\partial x}\right) .
$$

We consider the solution of Equation (38) on a uniformly spaced grid in $x$ defined by $x_{j}=(j-1) h$, with $j=1,2, \ldots, L$, where $h=1 /(L-1)$ is the grid spacing. Similarly, we consider discrete times $t_{n}=(n-1) \tau$, with $n=1,2, \ldots$, where $\tau$ is a constant time step. A suitable forward-time, centered-space (FTCS) discretization of Equation (38) is presented as follows (Press et al. 1992):

$$
\begin{aligned}
\frac{B_{j}^{n+1}-B_{j}^{n}}{\tau} \approx & \frac{4}{\pi^{2} r_{j}\left(1+r_{j}^{2}\right) h} \\
& \times\left(\frac{r_{j+\frac{1}{2}}}{1+r_{j+\frac{1}{2}}^{2}} D_{j+\frac{1}{2}}^{n} \frac{B_{j+1}^{n}-B_{j}^{n}}{h}\right. \\
& \left.-\frac{r_{j-\frac{1}{2}}}{1+r_{j-\frac{1}{2}}^{2}} D_{j-\frac{1}{2}}^{n} \frac{B_{j}^{n}-B_{j-1}^{n}}{h}\right),
\end{aligned}
$$

where $\quad B_{j}^{n}=B\left(x_{j}, t_{n}\right) \quad$ and $\quad D_{j}^{n}=D\left(B_{j}^{n}\right) \quad$ and where $r_{j}=\tan \left(\frac{\pi}{2} x_{j}\right)$. The diffusion coefficients at intermediate grid points may be approximated by

$$
D_{j \pm \frac{1}{2}} \approx D_{j \pm}=\frac{1}{2}\left(D_{j}^{n}+D_{j \pm 1}^{n}\right) .
$$

Equations (39) and (40) give the numerical scheme

$$
\begin{aligned}
B_{j}^{n+1}= & B_{j}^{n}+\frac{2}{\pi^{2} r_{j}\left(1+r_{j}^{2}\right)} \frac{\tau}{h^{2}} \\
& \times\left[\frac{r_{j+\frac{1}{2}}}{1+r_{j+\frac{1}{2}}^{2}} D_{j+}^{n}\left(B_{j+1}^{n}-B_{j}^{n}\right)\right. \\
& \left.-\frac{r_{j-\frac{1}{2}}}{1+r_{j-\frac{1}{2}}^{2}} D_{j-}^{n}\left(B_{j}^{n}-B_{j-1}^{n}\right)\right] .
\end{aligned}
$$

Equation (41) is an explicit prescription for time evolution at grid locations $j=2,3, \ldots, L-1$. For $j=1 \quad(r=0)$ the physical boundary condition is

$$
\left.\frac{\partial B}{\partial r}\right|_{r=0}=\left.\frac{2}{\pi\left(1+r^{2}\right)} \frac{\partial B}{\partial x}\right|_{r=x=0}=0
$$

for all times. Equation (42) may be enforced using a one-sided (forward) approximation to the derivative with respect to $x$ at $x=0$ :

$$
\left.\frac{\partial B}{\partial x}\right|_{x=0, t_{n+1}} \approx \frac{-3 B_{1}^{n+1}+4 B_{2}^{n+1}-B_{3}^{n+1}}{2 h}
$$

giving the update for the grid point $j=1$ :

$$
B_{1}^{n+1}=\frac{1}{3}\left(4 B_{2}^{n+1}-B_{3}^{n+1}\right) .
$$

This approach requires that the initial conditions satisfy Equation (44), i.e., $B_{1}^{1}=\frac{1}{3}\left(4 B_{2}^{1}-B_{3}^{1}\right)$.

For $j=L$, corresponding to $r=\infty$, we use the update

$$
B_{L}^{n+1}=B_{L}^{n}
$$

which together with the initial condition $B_{L}^{1}=0$ ensures that the field is zero at infinity.

Under the transformation (37), the total magnetic flux, defined by Equation (3), becomes

$$
\Phi_{0}=\pi^{2} \int_{0}^{1} r(x)\left[1+r(x)^{2}\right] B[r(x), t) d x .
$$


Equation (46) may be evaluated in our discrete version of the problem using the trapezoidal rule (Press et al. 1992):

$$
\Phi_{0}^{n}=\pi^{2} h \sum_{j=1}^{L} w_{j} r_{j}\left(1+r_{j}^{2}\right) B_{j}^{n},
$$

where $w_{j}=1$ for $j=2, \ldots, L-1$ and $w_{1}=w_{L}=\frac{1}{2}$. Equation (47) is used to check that flux is approximately conserved by the numerical solution.

A simple estimate of the stability condition for the method may be made as follows. We expect that an FTCS discretization of Equation (1) is stable at a given time step subject to the following (Press et al. 1992):

$$
\tau^{n} \leqslant \min _{j} \frac{\left(\Delta r_{j}\right)^{2}}{4 D_{j}^{n}}
$$

where $\Delta r_{j}$ is the grid spacing. (Strictly this requires a uniform grid in $r$.) From Equation (37) we have $\Delta r_{j} \approx \frac{\pi}{2}\left(1+r_{j}^{2}\right) h$ so Equation (48) becomes

$$
\tau^{n} \leqslant \frac{\pi^{2} h^{2}}{16} \min _{j} \frac{\left(1+r_{j}^{2}\right)^{2}}{D_{j}^{n}} .
$$

In the case of the model with a step dependence of $D$ on $B$ (Section 4) we can take

$$
\tau \leqslant \frac{\pi^{2} h^{2}}{16}\left(1+r_{1}^{2}\right)^{2} .
$$

Numerical experimentation suggests that Equation (50) provides a good estimate for the actual stability constraint.

\section{REFERENCES}

Barenblatt, G. I. 1952, Akad. Nauk SSSR. Prikl. Mat. Mekh., 16, 67

Barenblatt, G. I. 1996, Scaling, Self-Similarity, and Intermediate Asymptotics (Cambridge: Cambridge Univ. Press)

Bradshaw, S. J., \& Hartigan, P. 2014, ApJ, 795, 79

Brezis, H., \& Friedman, A. 1983, J. Math. Pures Appl., 62, 73

Chatterjee, P., Choudhuri, A. R., \& Petrovay, K. 2006, A\&A, 449, 781

Davenport, J. R. A., Hebb, L., \& Hawley, S. L. 2015, ApJ, 806, 212

Gafeira, R., Fonte, C. C., Pais, M. A., \& Fernandes, J. 2014, SoPh, 289, 1531

Giguere, M. J., Fischer, D. A., Zhang, C. X. Y., et al. 2016, ApJ, 824, 150

Goodman, T. R. 1958, Trans. ASME, 80, 335

Hill, J. M., \& Dewynne, J. N. 1987, Heat Conduction (Oxford: Blackwell Scientific)

Hurlburt, N., \& DeRosa, M. 2008, ApJL, 684, L123

King, J. R. 1990, JPhA, 23, 3681

Kitchatinov, L. L., Pipin, V. V., \& Rüdiger, G. 1994, AN, 315, 157

Krause, F., \& Rüdiger, G. 1975, SoPh, 42, 107

Künstler, A., Carroll, T. A., \& Strassmeier, K. G. 2015, A\&A, 578, A101

Landau, L. D., \& Lifshitz, E. M. 1987, Fluid Mechanics (Oxford: Elsevier)

Lepreti, F., Carbone, V., Abramenko, V. I., et al. 2012, ApJL, 759, L17

Litvinenko, Y. E. 2011, ApJL, 731, L39

Litvinenko, Y. E. 2015, JKAS, 48, 187

Litvinenko, Y. E., \& Wheatland, M. S. 2015, ApJ, 800, 130

Martínez Pillet, V., Moreno-Insertis, F., \& Vázquez, M. 1993, A\&A, 274, 521

Meyer, F., Schmidt, H. U., Wilson, P. R., \& Weiss, N. O. 1974, MNRAS, 169,35

Moreno-Insertis, F., \& Vázquez, M. 1988, A\&A, 205, 289

Pattle, R. E. 1959, QJMAM, 12, 407

Petrovay, K., Martínez Pillet, V., \& van Driel-Gesztelyi, L. 1999, SoPh, 188,315

Petrovay, K., \& Moreno-Insertis, F. 1997, ApJ, 485, 398

Petrovay, K., \& van Driel-Gesztelyi, L. 1997, SoPh, 176, 249

Press, W. H., Teukolsky, S. A., Vetterling, W. T., \& Flannery, B. P. 1992, Numerical Recipes in C: The Art of Scientific Computing (2nd ed; Cambridge: Cambridge Univ. Press)

Rempel, M., \& Cheung, M. C. M. 2014, ApJ, 785, 90

Rosenau, P. 1995, PhRvL, 74, 1056

Rüdiger, G., \& Kitchatinov, L. L. 2000, AN, 321, 75

Simon, G. W., \& Leighton, R. B. 1964, ApJ, 140, 1120 